\title{
A Monte Carlo Simulation Study of Electron Drift Velocities for Conditions with Electron Impact Ionization
}

\author{
Member Shigeru Yachi \\ (Hokkaido University) \\ Member Hiroaki Tagashira (Hokkaido University)
}

\begin{abstract}
A numerical calculation shows that the mean arrival time drift velocity $W_{m}$ and the average velocity $W_{v}$ of the electrons in isolated swarms assume very close values with common gases such as $\mathrm{Ar}, \mathrm{N}_{2}, \mathrm{CH}_{4}$ and $\mathrm{SiH}_{4}$. If $W_{m}=W_{v}$ is proved, there should be considerable influences on theoretical and experimental studies of electron swarms. The purpose of the present paper is to clarify if $W_{m}$ $=W_{v}$. By a study using a Monte Carlo simulation technique and deliberately arranged model cross sections, it is found that $W_{m} \neq W_{v}$ in general. A reason why $W_{m} \simeq W_{v}$ in real gases is given.
\end{abstract}

Key words : Electron drift velocity, Electron swarm, Ionization

\section{Introduction}

The electron drift velocity is one of the most important parameters which describe the behavior of electron swarms in gases ${ }^{(1)}$. Until some time ago, it had been believed that the electron drift velocity assumed a value depending upon the kind of gases and the value of $E / N$, where $E$ is the electric field and $N$ is the number density of gas molecules. In previous papers ${ }^{(2)(3)}$, however, it has been shown that the electron drift velocity depends not only on the kind of gases and on the value of $E / N$ but also on how it is defined. In the papers, it has also been shown that typical, theoretically clear-cut electron drift velocities may be defined either as the centerof-mass drift velocity $W_{r}$ of the electrons in an isolated swarm, or as the simple average velocity $W_{v}$ of the electrons in the swarm, or as the average velocity $V_{d}$ of the electrons in the steady-state Townsend experiment.

In a companion paper to the present paper ${ }^{(4)}$, one of the authors (H. T.) has pointed out that a drift velocity derived from another mode of observation of isolated electron swarms is important. The drift velocity is the mean arrival time drift velocity $W_{m}$ which is defined as the ratio of the difference between two positions, in the field direction, of observation of electron swarms to the difference between the average arrival times at the positions. The importance of $W_{m}$ comes from that in a most commonly used design of measurement of electron drift velocity, the mean transit time between two fixed positions in the field direction is measured and the drift velocity directly obtained from the ratio of the difference of the positions to the difference of the mean arrival times, or the transit time, is the mean arrival time drift velocity $W_{m}$.

One of the interesting problems concerning $W_{m}$ is that the numerical value of $W_{m}$ is found to be very close to that of $W_{v}$, the drift velocity defined as the average velocity of the electrons in an isolated swarm, for conditions even with considerable electron impact ionization. An example is shown in Table 1 for $\mathrm{Ar}^{(2)(5)}$. Note that when there is no ionization, nor electron attachment, $W_{m}, W_{v}, W_{r}$ and $V_{d}$ as well, should have the same value ${ }^{(2)}$.

In the table, the value of $W_{m}$ is calculated by using two approximate formulae ${ }^{\langle 4\rangle}$ as below.

$$
W_{m} \S=W_{r}-2 \alpha_{T} D_{L}
$$


Table 1. Comparison ef $W_{m}$ and $W_{v}$ (Ar).

\begin{tabular}{l|c|c|c|c|c|c}
\hline \multirow{2}{*}{ Parameter } & \multicolumn{6}{|c}{$E / N(\mathrm{Td})$} \\
\cline { 2 - 7 } & 85 & 141 & 212 & 283 & 424 & 566 \\
\hline$W_{r}\left(\mathrm{~cm}^{*} \mu \mathrm{s}^{-1}\right)$ & 7.34 & 12.0 & 17.9 & 23.9 & 36.5 & 50.5 \\
$\alpha_{r}\left(\mathrm{~cm}^{-1}\right)$ & 0.231 & 0.669 & 1.29 & 1.92 & 3.16 & 4.30 \\
$D_{L}\left(\mathrm{~cm}^{2} \cdot \mu \mathrm{s}^{-1}\right)$ & 1.01 & 1.06 & 1.14 & 1.24 & 1.49 & 1.87 \\
$D_{3}\left(\mathrm{~cm}^{3} \cdot \mu \mathrm{s}^{-1}\right) *$ & & 0.036 & & 0.028 & & 0.049 \\
$W_{m}\left(\mathrm{~cm}^{*} \mu \mathrm{s}^{-1}\right) \S$ & 6.87 & 10.58 & 14.96 & 19.14 & 27.1 & 34.48 \\
$\Delta W(\%) \S$ & 0.15 & -0.19 & -0.93 & -1.85 & -4.24 & -7.56 \\
$W_{m}\left(\mathrm{~cm}^{*} \mu \mathrm{s}^{-1}\right) \S$ & & 10.62 & & 19.45 & & 36.87 \\
$\Delta W(\%) \S \S$ & & 0.19 & & -0.26 & & -1.15 \\
$W_{v}\left(\mathrm{~cm}^{*} \mu \mathrm{s}^{-1}\right)$ & 6.86 & 10.6 & 15.1 & 19.5 & 28.3 & 37.3 \\
\hline $\mathrm{A}$ & & &
\end{tabular}

A gas number density $N=3.54 \times 10^{16} \mathrm{~cm}^{-3}$ is assumed.

$W_{m} \S=W_{r}-2 \alpha_{T} D_{L}, W_{m} \S \S=W_{r}-2 \alpha_{r} D_{L}+3 \alpha_{T}^{2} D_{3} ; \Delta W(\%)=100\left(W_{m}-\right.$ $\left.W_{v}\right) / W_{v}$

The values of the parameters are taken from Ref. (2) except the values*, which are taken from Ref. (5). In this reference, the parameters are deduced from the same cross sections as in Ref. (2).

$$
W_{m} \S \S=W_{r}-2 \alpha_{T} D_{L}+3 \alpha_{T}^{2} D_{3}
$$

Here, $\alpha_{T}$ is the Townsend first ionization coefficient, $D_{L}$ is the longitudinal diffusion coefficient, which is the coefficient of the second order gradient term in the field direction of the electron number density, and $D_{3}$ is the coefficient of the third order gradient term in the field direction of the electron number density, in the continuity equation of electrons for electron swarms ${ }^{(2)(6)(7)}$. The expression of $W_{m}$ in Eqs ( 1 ) and (2) comes from the result of the companion paper $^{(4)}$ in which an exact expression of $W_{m}$ is obtained in an infinite series form. The expression in Eq. (1) is derived by taking the first two terms, and that in Eq. (2) by taking the first three terms, of the exact expression, and therefore the expression in Eq. (2) is expected to be more exact than that in Eq. (1) since the magnitude of each of the terms decreases very rapidly as the order of the terms increases under the conditions considered here.

In the table, some electron swarm parameters which are necessary to calculate $W_{m}$ are also listed for reference.

It is seen in Table 1 that the percentage difference between the mean arrival time drift velocity $W_{m}$ and the drift velocity defined as the average $W_{v}$ is remarkably small even at high $E / N$ values at which ionization is appreciable judging from the value of the ionization coefficient $\alpha_{T}$.

The same calculation as given in Table 1 has also been performed for $\mathrm{N}_{2}, \mathrm{CH}_{4}$ and $\mathrm{SiH}_{4}$ and found that the difference between $W_{m}$ and $W_{v}$ are very small, only a few percent even at $E / N=566 \mathrm{Td}$.

In all the gases, the difference between $W_{r}$, the center-of-mass drift velocity, and $W_{v}$ at $E / N=$ $566 \mathrm{Td}$, at which ionization is considerable, differred more than $10 \%$ which may be regarded as large enough to prove the difference even with numerical calculations. Since numerical calculations are in general subject to errors due to approximation employed in theory and also due to numerical computation, it is suspected that the two drift velocities $W_{m}$ and $W_{v}$ are essentially the same drift velocity. Therefore, considerable efforts have been made to analytically prove that $W_{m}=W_{v}$. However, until now, it remains difficult to prove, or disprove, $W_{m}=W_{\nu}$.

The purpose of the present paper is to solve this problem by using a Monte Carlo simulation technique. This technique is employed here since it does not have any approximation like truncation of a series. One of the weak point of the technique is a relatively large numerical scatter in the result. However, this may be overcome firstly by using enough number of electrons to reduce the scatter to or less than a reasonable level and secondly by using fictitious cross sections with which the difference between $W_{m}$ and $W_{v}$, if any, is large enough to overcome the numerical scatter.

\section{Cross sections and simulation method}

\subsection{Collision cross sections}

The main purpose of the present paper is to judge if the inductively raised hypothesis $W_{m}=W_{v}$ is generally correct. As mentioned in the previous section, the numerical examples of $W_{m}$ and $W_{v}$ for common gases $\mathrm{Ar}, \mathrm{N}_{2}$ and $\mathrm{CH}_{4}$ and a gas, $\mathrm{SiH}_{4}$, which has become very popular recently for plasma CVD applications, all strongly suggest that $W_{m}$ might be equal to $W_{v}$. From a standpoint such that we prove $W_{m}=W_{v}$, we should collect as many data as possible to support $W_{m}=W_{v}$, but if we choose the opposite standpoint to disprove $W_{m}=W_{v}$, then it is a matter of trying to find at least one case against $W_{m}$ $=W_{v}$. Taking the latter action is chosen here since it appears simpler. However, if the truth is that 


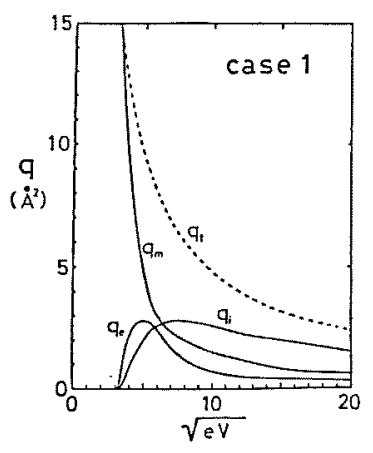

$q_{m}, q_{e}, q_{i}$ and $q_{t}$ are respectively the momentum trasfer, excitation, ionization and total cross sections. The threshold of $q_{e}(=9.0 \mathrm{eV})$ is lower than that of $q_{i}(=10.24 \mathrm{eV})$.

Fig. 1. Electron collision cross sections against electron energy $\varepsilon$ for model gas 1 .

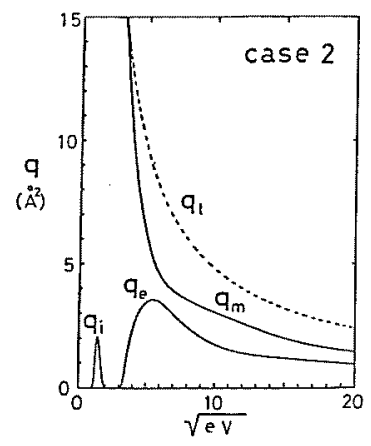

$q_{m}, q_{e}, q_{i}$ and $q_{\mathrm{t}}$ are the same as Fig. 1. The threshold of $q_{e}(=9.0 \mathrm{eV})$ is higher than that of $q_{r}(=1.0 \mathrm{eV})$.

Fig. 2. Electron collision cross sections against electron energy $\varepsilon$ for model gas 2 .

$W_{m} \neq W_{v}$, then how the examples mentioned above, with which $W_{m}$ is very much likely to be equal to $W_{v}$, are explained? A possibility is that the threshold of ionization $\varepsilon_{i}$ is located at a higher electron energy than that of excitation $\varepsilon_{e}$. Although a theoretical explanation is difficult, it is at least a common feature of the gases. It has been decided, therefore, to add such a set of cross sections in which $\varepsilon_{i}<\varepsilon_{e}$ for test. There should be no such single gases, with $\varepsilon_{i}<\varepsilon_{e}$, therefore such a choice of collision cross section is unreal. However, since the model and theory of electron swarms does not necessarily exclude such a case in which $\varepsilon_{i}<\varepsilon_{e}$ in the process of its construction and also since such a

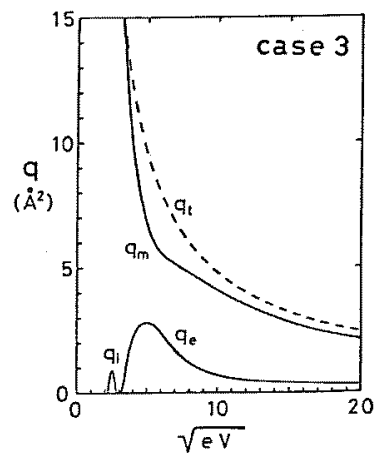

$q_{m}, q_{e}, q_{i}$ and $q_{t}$ are the same as Fig. 1. The threshold of $q_{e}(=9.0 \mathrm{eV})$ is higher than that of $q_{i}(=4.8 \mathrm{eV})$.

Fig. 3. Electron collision cross sections against electron energy $\varepsilon$ for model gas 3 .

gas with $\varepsilon_{i}<\varepsilon_{e}$ may be "approximately created" by mixing gases, a choice of such a set of cross sections with which $\varepsilon_{i}<\varepsilon_{e}$ may be allowed and not too unreal.

The cross sections used in the present calculation are given in Figs $1 \sim 3$. The inelastic processes, excitation and ionization here, are represented by the respective single cross sections for simplicity. The total cross section, this being the sum of the elastic and inelastic cross sections, are chosen so that the collision frequency is constant regardless of electron energy. This choice simplifies the procedure of judgement of collision in the computational routine of Monte Carlo simulation ${ }^{(8)}$.

The thresholds for excitation and ionization of the cross sections in Fig. 1 are chosen respectively $9.0 \mathrm{eV}$ and $10.24 \mathrm{eV}$. These cross sections are shaped more or less similar to real ones when compared to those in Figs. 2 and 3.

The position and shape of the ionization cross section for Figs. 2 and 3 are chosen to be rather unreal since $\varepsilon_{i}<\varepsilon_{e}$ and the width of the cross section is very narrow. The ionization cross section $q_{i}$ in Fig. 2 has the threshold $\varepsilon_{i}$ at $1 \mathrm{eV}$ and has a width of $3 \mathrm{eV}$, while $q_{i}$ in Fig. 3 spreads from $4.8 \mathrm{eV}$ to $7.8 \mathrm{eV}$. The cases for Figs. 2 and 3 may appear qualitatively similar. However, it has been deliberately arranged so that $\varepsilon_{i}<\bar{\varepsilon}$ for Fig. 2 and $\varepsilon_{i} \simeq \bar{\varepsilon}$ for Fig. 3 where $\bar{\varepsilon}$ is the electron mean energy.

The calculation is extended to another case in which $q_{i}$ in Fig. 1 is treated in the same way as $q_{e}$ so 
that there is no ionization, thus making a check of the calculation since in this case all the drift velocities should assume the same value ${ }^{(2)}$.

\subsection{Method of calculation}

A standard Monte Carlo technique has been employed $^{(8)}$. Briefly, the flight time $\tau$ of electrons is generated simply as follows.

$$
\tau=-\nu^{-1} \ln (1-\xi)
$$

where $\nu$ is the collision frequency, taken constant in the present work, and $\xi$ is a uniform random number between 0 and 1 . During $\tau$, the electron enjoys a free flight under the action of a uniform electric field obeying Newton's law of motion. When a collision occurs, the kind of collision is judged in such a way that the collision of a kind occurs with a probability which is proportional to the magnitude of the cross section of the kind. All the scatterings after collisions are assumed isotropic. After ionization collisions, the remaining energy of the incident electron is shared to the two electrons at a ratio of 0.2 to 0.8 . All the electrons generated after ionization collisions are traced as well as the initial electrons. The electron drift velocities and other swarm parameters are sampled obeying their definitions ${ }^{(8)}$. To avoid the electron energy non-equilibrium effect as much as possible, the velocity distribution in equilibrium is obtained beforehand, and the initial electrons are released with the velocity distribution at a position at time $t=0$.

\section{Results and discussion}

The calculation is done for four cases. In case 1 , the collision cross sections for model gas 1 as shown in Fig. 1 are employed and the simulation is performed at an $E / N=354 \mathrm{Td}$.

The average velocity $W_{v}$ of the electrons in an isolated swarm is sampled and shown in Fig. 4. As mentioned at the end of the previous section, the initial electrons are started with the equilibrium distribution and this is why the time required for equilibrium velocity is practically zero in Fig. 4. A reasonably accurate value may be sampled for $W_{v}$ as seen from the figure.

In Fig. 5 is shown the distribution $n(z, t)$ of the arrival time of electrons at positions $z=0.2,0.5$, and

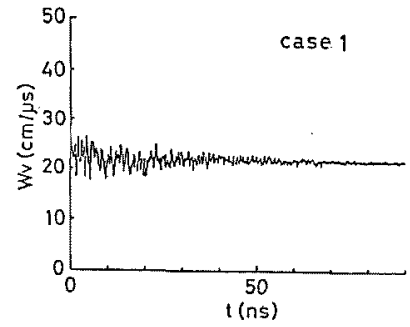

Fig. 4. Behavior of the average electron velocity $W_{v}$ of isolated swarm for case 1 (=model gas 1 at $E / N=354 \mathrm{Td}$ ). The fluctuation diminishes as the number of electrons increases.

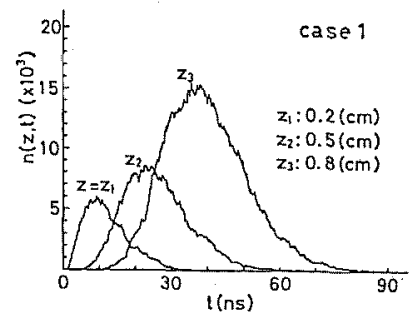

Fig. 5. Distribution of arrival time at positions in the field direction for case 1. A thousand initial electrons are injected at position $z=0$ at time $t=0$.

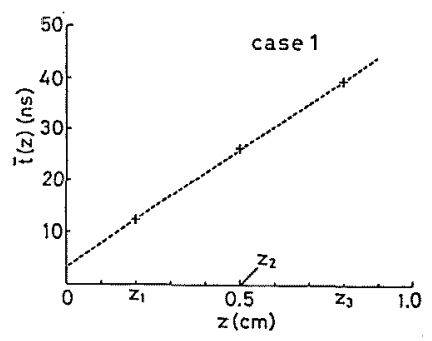

Fig. 6. Mean arrival time $\bar{t}$ as a function of position $z$ for case 1 as derived from Fig. 5. Mean arrival time drift velocity $W_{m}$ is obtained from the inverse of the slope of the straight line.

$0.8 \mathrm{~cm}$ in the field direction for case 1 . The mean arrival time $\bar{t}$ is calculated from the distribution and plotted in Fig. 6 . The points lie well on a straight line and the mean arrival time drift velocity $W_{m}$ for case 1 is obtained as the inverse of the slope of the line.

Fig. 7 shows the position of the center-of-mass of 


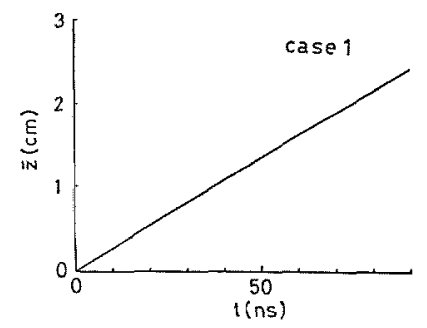

Fig. 7. Variation of the center-of-mass $\bar{z}$ of an isolated electron swarm as a function of $t$ for case 1 . The center-of-mass drift velocity $W_{\tau}$ is obtained as the slope of the straight line.

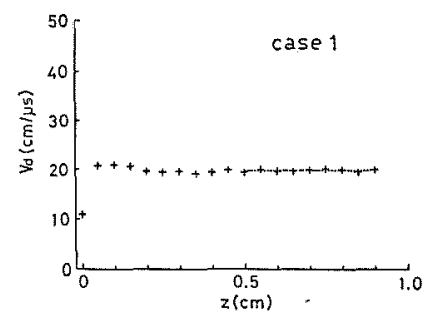

Fig. 8. Variation of the average electron velocity $V_{d}$ for the steady-state Townsend condition for case 1 . The fluctuation tends to diminish with $z$.

an isolated swarm $\bar{z}$ as a function of time for case 1. It is seen that the position lies practically on a straight line and from its slope the center-of-mass drift velocity is obtained. Similarly in Fig. 8 the average velocity $V_{d}$ of the electrons for the steady. state Townsend condition is sampled as a function of the position.

Figs 4, 6 8 show that the present simulation can determine $W_{\nu}, W_{m}, W_{r}$ and $V_{d}$ at a reasonable accuracy.

The calculation is performed also for cases 2,3 and 4 .

In cases 2 and 3 , the cross sections given in Figs 2 and 3 , respectively, are used. For case 4 , the calculation is performed by using the set of cross sections in Fig. 1 in which $q_{i}$, the ionization cross section, is treated also as $q_{e}$, an excitation cross section, so that there is no ionization cross section and two excitation cross sections. The $E / N$ is assumed to be $354 \mathrm{Td}$ in all the cases.
Table 2. Comparison of $W_{m}$ and $W_{v}$ (model gases).

\begin{tabular}{|c|c|c|c|c|}
\hline & \multicolumn{4}{|c|}{$E / N=354 \mathrm{Td}$} \\
\hline & case 1 & case 2 & case 3 & case 4 \\
\hline$W_{r}\left(\mathrm{~cm} \cdot \mu \mathrm{s}^{-1}\right)$ & 26.8 & 18.8 & 21.5 & 21.8 \\
\hline$\alpha_{T}\left(\mathrm{~cm}^{-1}\right)$ & 2.7 & 4.2 & 3.2 & 0.0 \\
\hline$D_{L}\left(\mathrm{~cm}^{2} \cdot \mu \mathrm{s}^{-1}\right)$ & 0.95 & 0.73 & 0.69 & 0.83 \\
\hline$D_{3}\left(\mathrm{~cm}^{3} * \mu \mathrm{s}^{-1}\right)$ & 0.03 & 0.03 & 0.02 & 0.03 \\
\hline$R_{i}\left(\mu \mathrm{s}^{-1}\right)$ & 66.7 & 69.0 & 62.3 & 0.0 \\
\hline$E(e V)$ & 6.34 & 6.46 & 6.29 & 6.92 \\
\hline$W_{m}\left(\mathrm{~cm} \cdot \mu \mathrm{s}^{-1}\right)$ & 22.0 & 14.3 & 17.4 & 21.8 \\
\hline$\Delta W(\%)$ & 1.38 & -33.80 & -19.44 & 0.0 \\
\hline$W_{m}\left(\mathrm{~cm} \cdot \mu \mathrm{s}^{-1}\right)^{*}$ & 21.7 & 12.6 & 17.0 & 21.8 \\
\hline$\Delta W(\%)^{*}$ & 0.0 & -41.67 & -21.30 & 0.0 \\
\hline$W_{m}\left(\mathrm{~cm} \cdot \mu \mathrm{s}^{-1}\right)^{* *}$ & 22.1 & 14.2 & 17.7 & 21.8 \\
\hline$\Delta W(\%)^{* *}$ & 1.84 & -34.26 & -18.06 & 0.0 \\
\hline$W_{v}\left(\mathrm{~cm} \cdot \mu \mathrm{s}^{-1}\right)$ & 21.7 & 21.6 & 21.6 & 21.8 \\
\hline$V_{d}\left(\mathrm{~cm} \cdot \mu \mathrm{s}^{-1}\right)$ & 19.4 & 18.7 & 19.7 & 21.8 \\
\hline \multicolumn{5}{|c|}{$\begin{array}{l}\text { A gas number density } N=3.54 \times 10^{16} \text { is assumed. } \\
W_{m} \text { is directly sampled by Monte Carlo simulation as other values } \\
\text { without*. } \\
W_{m}^{*}=W_{r}-2 \alpha_{\tau} D_{L} ; W_{m}^{* *}=W_{r}-2 \alpha_{r} D_{L}+3 \alpha_{T}^{2} D_{3} ; \Delta W(\%)=100\left(W_{m}-\right. \\
\left.W_{v}\right) / W_{v}\end{array}$} \\
\hline
\end{tabular}

The results, including those for case 1 as well, are shown in Table 2.

It is seen in the table that $W_{m}$ and $W_{v}$ for case 1 assume very similar values. The difference is less than $2 \%$. From the fluctuation in simulation as seen in Figs $4 \sim 8$, the difference may be judged to lie within the error of the Monte Carlo calculations. Therefore, it is difficult to say whether $W_{m}=W_{v}$ or not from the result of case 1 .

If the results of simulation for case 2 are looked at, however, it is now clear that $W_{m} \neq W_{v}$. The difference $\Delta W$ amount to as large as or more than $30 \%$. A similar result is obtained with case 3 . The difference $\Delta W$ is about $20 \%$. The differences are judged far greater than the computational error. These two cases demonstrate that $W_{m} \neq W_{v}$ in general.

Case 4 shows that $W_{m}=W_{v}=W_{r}=V_{d}$ if there is no ionization. This is a conclusion analytically drawn ${ }^{(2)}$, and this case is an indication that the present simulation is performed properly.

It is interesting to note that the electron mean energy $\bar{\varepsilon}$ and ionization threshold $\varepsilon_{i}$ are in such a relation as $\bar{\varepsilon}<\varepsilon_{i}$ in case 1 . Then, ionization occurs considerably more in front of an isolated swarm 
than in the rear, and this results in $W_{v}<W_{r}{ }^{(2)}$. Note that the electron energy is higher in front than in tail of an isolated swarm since the flow of electron current due to the action of the electric field and due to diffusion add in front whereas they subtract in the rear $^{(2)}$. Since $W_{m}<W_{r}$ as seen from Eq. (1) or (2), because the second and particularly third term are usually smaller than the first on the right hand side of those equations, there is certainly a possibility that $W_{m} \simeq W_{v}$ in case 1 . If there is an excitation cross section, the threshold of which $\varepsilon_{e}$ is lower than $\varepsilon_{i}$, the electron energy is lost to excitation and the electron mean energy does not increase easily. This mechanism tends to keep $\dot{\varepsilon}<\varepsilon_{i}$ and so $W_{v}<W_{r}$. Therefore, what is essential is the mutual position of $\bar{\varepsilon}$ and $\varepsilon_{i}$, but $\varepsilon_{e}<\varepsilon_{i}$, as in case 1 and in real gases, assists to keep $\bar{\varepsilon}<\varepsilon_{i}$, which tends to give $W_{v}<W_{r}$ and when combined with $W_{m}<W_{r}, W_{m} \simeq W_{v}$, as has been discussed.

In case 2 , it is seen that $\bar{\varepsilon}>\varepsilon_{i}$. This leads to $W_{v}$ $>W_{r}$ since ionization occurs more frequently in the rear than in front of an isolated swarm. The relationship between $W_{m}$ and $W_{r}$ is $W_{m}<W_{r}$ as seen in Eqs. (1) and (2). Therefore, $W_{m}<W_{v}$ is brought about.

The cross sections for case 3 are chosen so that the $q_{i}$ assumes significant values at around $\bar{\varepsilon}$, as seen from Fig. 3 and Table 2. Namely, $\varepsilon_{i} \simeq \bar{\varepsilon}$. This results in more or less a balanced ionization within a swarm which leads to $W_{v} \simeq W_{r}$. Since $W_{m}<W_{r}$ by Eq. (1) or (2), $W_{m} \neq W_{v}$ in case 3 as well.

A check of accuracy of Eqs. (1) and (2) may be made by comparing $W_{m}$ with $W_{m}{ }^{*}$ or $W_{m}{ }^{* *}$. It is clear that $W_{m}{ }^{* *}$ calculated from Eq. (2) is in better agreement with $W_{m}$ which is directly sampled in Monte Carlo simulation and therefore more accurate. This may be an indication that the formula for the accurate mean arrival time is correct.

\section{Conclusions}

The purpose of the present paper is to prove, or disprove, the seemingly holding relationship $W_{m}=$ $W_{v}$, where $W_{m}$ is the mean arrival time drift velocity and $W_{v}$ is the average velocity of the electrons in an isolated swarm. Several examples of real gases seemed to suggest that $W_{m}=W_{v}$.
It has been shown by using a fictitious, but deliberately arranged, sets of electron-gas molecule collision cross sections that in general $W_{m} \neq W_{v}$. A reason has been given why $W_{m} \simeq W_{v}$ in real gases. It has also been shown that the expression of $W_{m}$ given by Eq. (2), which includes the first three terms, is more accurate than $W_{m}$ given by Eq. (1) which retains the first two terms.

\section{Acknowledgement}

The authors wish to thank Professor Y. Sakai, Drs. K. Kitamori and Y.Ohmori for interest and much helpful discussion.

(Manuscript received June 5,

'90, revised Aug. 1, '90)

\section{References}

(1) L. G. H. Huxley \& R. W. Crompton: Diffusion and Drift of Electrons in gases, Chap. 4 (1974) Wiley, New York

(2) H. Tagashira, Y.Sakai \& S. Sakamoto: "The development of electron avalanches in argon at high $E / N$ values: II. Boltzmann equation analysis", J. Phys. D: Appl. Phys., 10, 1051 (1977)

(3) H. Tagashira: "Development of Electron avalanches at High Electric Fields", Proc. XV Int. Conf. on Phenon. in Ionized Gases (BSSR Academy of Sciences, Minsk) Pt. 3 (Invited Papers), p. 377 (1981)

(4) H. Tagashira: "An exact expression of the mean arrival time drift velocity of electron swarms in gases" Trans. IEE Japan, Vol. III-A, No. 3(1991-3)

(5) K. Kitamori, H. Tagashira \& Y. Sakai: "Development of electron avalanches in argon - an exact Boltzmann equation analysis", J. Phys. D: Appl. Phys., 13, 535 (1980)

(6) K. Kumar, H. R. Sukullerud \& R. E. Robson: "Kinetic Theory of Charged Particles in Neutral Gases", Austr. J. Phys, 33, 343 (1980)

(7) J. H. Parker Jr. \& J. J. Lowke: "Theory of Electron Diffusion Parallel to Electric Fields I. theory", Phys. Rev., 181. 290 (1969)

(8) "Gas Discharge Simulation Techniques," Technical Report (Pt. II), No. 140, IEE, Japan, Tokyo, Section 3.1.3 (1982)

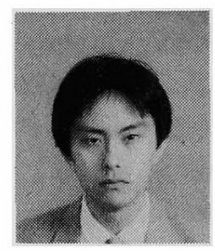

\section{Shigeru Yachi (Member)}

He was born on Nov. 29, 1961. He finished Master's Course, Division of Electrical Engineering, Graduate School of Engineering, Hokkaido University in March 1986. He is now with Material Research Laboratory, Mitsubishi Electric Company.

He is a member of The Japan Society of Applied Physics and The Institute of Electronics, Information and Communication Engineers. 


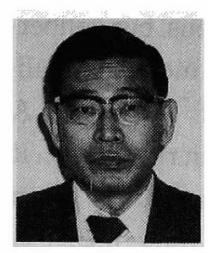

Hiroaki Tagashira (Member)

Engineering, Hokkaido University. He received $\mathrm{Ph}$ D. from Liverpool University in 1967 and D. Eng.

He was born on Jan. 13, 1933. He finished Master's Course, Division of Electrical Egineering, Graduate School of Engineering, Hokkaido University in March 1960 . He became Lecturer in 1961, Assistant Professor in 1962 and Professor in 1973 at the Department of Electrical from Hokkaido University in 1980 . He is a member of International Scientific Committee of the International Conference on Phenomena in Ionized Gases. $\mathrm{He}$ is a member of Illumination Engineering Society of Japan, Physical Society of Japan, and The Japan Society of Applied Physics. 